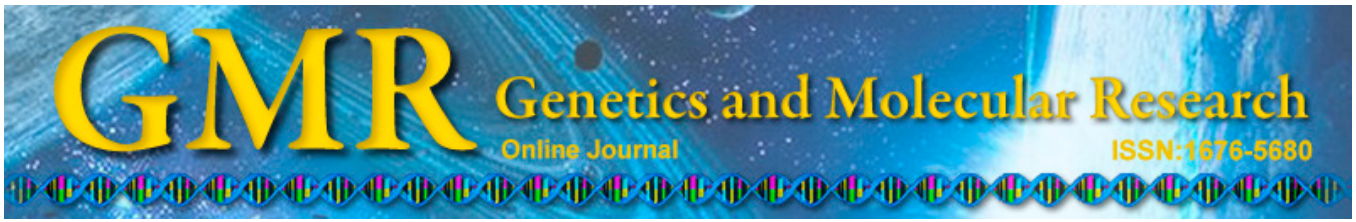

\title{
Influence of the $D C C$ gene on proliferation and carcinoembryonic antigen expression in the human colorectal cancer cell line SW1116
}

\author{
H.W. Jiang, J. Wang, H.J. Li, J.K. Peng, X.P. Gao and F. Chen \\ Department of Gastrointestinal Surgery, Inner Mongolia People's Hospital, \\ Hohhot, China \\ Corresponding author: H.W. Jiang \\ E-mail: jianghongwei_b@163.com / Jhww2009@163.com
}

Genet. Mol. Res. 14 (3): 10273-10280 (2015)

Received December 18, 2014

Accepted May 15, 2015

Published August 28, 2015

DOI http://dx.doi.org/10.4238/2015.August.28.12

\begin{abstract}
This study investigated the effects of stable transfection of the exogenous wild-type $D C C$ gene on growth of the human colorectal carcinoma cell line SW1116 in vitro. The DCC gene was amplified from normal human colon tissue by reverse transcription-polymerase chain reaction and used to construct a recombinant expression plasmid, pcDNA3.1(+)-DCC. DCC-negative SW1116 cells were transfected with pcDNA3.1(+)-DCC. Cell viability was tested by the methyl thiazolyl tetrazolium (MTT) assay. Immunofluorescence staining was used to determine the effects of pcDNA3.1(+)-DCC on carcinoembryonic antigen (CEA) expression in transfected cells. The number of cells in the population transfected with pcDNA3.1(+)-DCC was lower than in that transfected with the control pcDNA3.1(+) plasmid or in normal cells $(t 1=3.645, \mathrm{P} 1<0.05$, $t 2=3.132, \mathrm{P} 2<0.05)$ at $3-6$ days after transfection, and the proliferation rate of pcDNA3.1(+)-DCC transfected cells was also lower $(t 1=2.134$, $\mathrm{P} 2<0.05 ; t 2=2.736, \mathrm{P} 2<0.05)$. The total viability of pcDNA3.1 $(+)-$ DCC transfected cells was lower than that of normal cells $(t 1=3.053, \mathrm{P} 1$ $<0.05)$ at 2-6 days after transfection, and of control-transfected cells $(t 2=$ $2.816, \mathrm{P} 2<0.05)$ after $2,4,5$, and 6 days. The population of pcDNA3.1(+)-
\end{abstract}


DCC transfected colored of green fluorescent cells and their fluorescent intensities were lower than those of control-transfected and normal cells. Therefore, the transfected $D C C$ gene can suppress cell proliferation and lead to downregulation of CEA expression in SW1116 cells, which might weaken its infiltration and metastasis abilities.

Key words: Colorectal neoplasms; Transfection; $D C C$ gene

\section{INTRODUCTION}

Cell signal transduction is related to cell differentiation and growth as well as to proliferation and apoptosis and also has been shown to have a relationship with angiogenesis; this latter function has aroused attention as it is associated with the incidence of tumors (Defacque et al., 2000). The keys to gene therapy have been proposed to be the cloning of the target gene, i.e., which needs to be transferred to effect therapeutic response, and the construction of a eukaryotic expression vector that facilitates high level expression of the target gene, to ensure that the expressed product has biological activity (Dard et al., 2001). In this study, DCC was selected as the target gene for cloning and analysis as it represents a highly deactivated tumor suppressor gene in colorectal cancer, in order to find new ways and methods to treat colorectal cancer with gene therapy. A functional fragment of the $D C C$ gene was isolated from healthy colon tissue and amplified, and used to construct the pcDNA3.1(+)-DCC recombinant vector. The expression of the DCC gene in the human colorectal cancer cell line SW1116 and its consequences to cell activities was studied using reverse transcription-polymerase chain reaction (RT-PCR), the methyl thiazolyl tetrazolium (MTT) assay, and by immunofluorescence.

\section{MATERIAL AND METHODS}

\section{Cell line and reagents}

The human colorectal cancer cell line SW1116 was obtained from the Shanghai Institutes for Biological Sciences. The pMD18-T plasmid and Escherichia coli competent cells JM109 (stored in Department of Gastrointestinal Surgery, Inner Mongolia People's Hospital) were used in this study, and the pcDNA3.1(+) plasmid was purchased from TaKaRa (Dalian, China).

\section{Methods}

The primers were designed according to the mRNA sequence of the DCC gene (accession No. NM_005215.3) reported in GenBank and in published references (Li et al., 2006), and were synthesized by TaKaRa. The sequences of the $D C C$ primers are as follows: forward: 5'-AAG CTT CCG CCA CCA TGA CAG TCT TGG TTC CGC CAT G-3', and reverse: 5'- C TC TCT CTC GAG CTA ACT GGA GCT TGG GAT AGC A GGC-3', in which AAGCTT represents the HindIII restriction site, and CTCGAG represents the XhoI restriction site. The sequence CCG CCA CCA TG reflects the Kozak consensus sequence.

\section{Sample collection and extraction of total RNA}

Total RNA was extracted from normal colon tissues using RNAiso Plus (TaKaRa). 
Samples were obtained from the Department of Gastrointestinal Surgery of the People's Hospital in the Inner Mongolia Autonomous Region from April to June, 2010. Written approval for the study was obtained from the People's Hospital. All tissues were obtained with premortem consent in accordance with the laws and regulations in place in the various jurisdictions. Samples were pathologically diagnosed as positive for colorectal adenoma. Fresh specimens were collected at a $10 \mathrm{~cm}$ distance from the tumor, immediately placed at $-80^{\circ} \mathrm{C}$, then preserved in liquid nitrogen.

\section{Reverse transcription-polymerase chain reaction (RT-PCR)}

RT-PCR was conducted using the PrimeScript One Step RT-PCR Kit Ver.2 (TaKaRa). The protocol followed the manufacturer instructions. PCR amplification system $(50 \mu \mathrm{L}$ reaction system) was followed: RT-PCR conditions were followed: Prime enzyme mix $2 \mu \mathrm{L}$, buffer (Dye Plus) $25 \mu \mathrm{L}$, upstream primer $(20 \mu \mathrm{M}, 1 \mu \mathrm{L})$, downstream primer $(20 \mu \mathrm{M}, 1 \mu \mathrm{L})$, template RNA $\left(0.5 \mu \mathrm{L}\right.$ ), RNase free $\mathrm{dH}_{2} \mathrm{O}$ (to $50 \mu \mathrm{L}$ ). Three-step PCR including predegeneration at $94^{\circ} \mathrm{C}$ for 2 min; degeneration at $94^{\circ} \mathrm{C}$ for $30 \mathrm{~s}$, annealing at $60^{\circ} \mathrm{C}$ for $30 \mathrm{~s}$, extension at $72^{\circ} \mathrm{C}$ for $1 \mathrm{~min}$, totally 30 cycles.

\section{Amplification of the DCC functional fragment}

The DCC gene was amplified from human normal colon tissues using RT-PCR, as described above.

\section{Construction and validation of the pMD18-T-DCC recombinant vector}

The amplified PCR product of the $D C C$ gene functional fragment was recovered from an agarose gel, and ligated with the pMD18-T vector (T4 DNA ligase Kit, TaKaRa), then transformed into JM109 competent bacteria. Monoclonal colonies were picked, and positive recombination was identified by enzyme digestion. DNA sequencing was conducted (Life Technologies, Thermo Fisher Scientific Inc.) on the inserted fragments and their accuracy was confirmed by sequence alignment with the mRNA sequence of the DCC gene (accession No. NM_005215.3) as reported in GenBank. The alignment results showed complete homology to the reported sequence.

\section{Construction of the pcDNA3.1(+)-DCC recombinant vector}

The pMD18-T-DCC plasmid confirmed by sequence analysis and the pcDNA3.1(+) plasmid were double digested using the $\mathrm{XhoI}$ and HindIII restriction enzymes, and the concentrations of the $D C C$ gene and vector fragments were quantified and the fragments recovered using $1 \%$ agarose gel electrophoresis. Ligation reactions of the $D C C$ gene fragments and $\mathrm{pcD}-$ NA3.1(+) were conducted using T4 DNA ligase (TaKaRa), and the products were transformed into $E$. coli JM109 competent bacteria. Monoclonal colonies were picked, and positive recombinants were identified by enzyme digestion. Two colonies were selected and identified using double digestion with XhoI and HindIII, which showed a target band ( $335 \mathrm{bp}$ ) between 250 and 500 bp. This result suggested that the two colonies were each positive for recombinant pcDNA3.1(+)DCC. The pcDNA3.1(+)-DCC JM109 and pcDNA3.1(+) JM109 plasmids were cultured at a large scale, and DNA was extracted for use in subsequent cell transfections. High concentrations of the pcDNA3.1(+)-DCC $(1.55 \mu \mathrm{g} / \mu \mathrm{L})$ and pcDNA3.1(+) $(1.12 \mu \mathrm{g} / \mu \mathrm{L})$ plasmids were obtained. 


\section{Detection of the activities of SW1116 cells transfected with pcDNA3.1(+)-DCC}

The growth of SW1116 cells transfected with pcDNA3.1(+)-DCC was observed. Cells were split into a confluent plate 1:20 the day before the transfection. Added $20 \mu \mathrm{g}$ DNA to $1 \mathrm{~mL}$ 2X HEPES buffer (280 mM NaCl, $50 \mathrm{mM}$ HEPES, $1.5 \mathrm{mM} \mathrm{NaPO}_{4}$, pH 7.1). Added $1 \mathrm{~mL} 0.25 \mathrm{M}$ $\mathrm{CaCl} 2$ dropwise shaking vigorously after each drop. Allowed precipitate to form $30 \mathrm{~min}$ at room temperature. Vortex and added to $10 \mathrm{~cm}$ cell culture plate ( $\sim 30-50 \%$ confluent). Incubated 20 min at $37^{\circ} \mathrm{C}$. Mixed once during this time. Added $7 \mathrm{~mL}$ of warm media and incubated overnight. Removed media, glycerol shocked with $12.5 \%$ sterile glycerol in medium, and incubated $1 \mathrm{~min}$ at room temperature. Removed glycerol solution and rinsed cells twice with PBS. Added medium with serum (DMEM $+10 \% \mathrm{FBS}$ ). Cells were detached with $0.25 \%$ trypsin and counted at $1,2,3,4$, 5 , and 6 days after transfection, and a growth curve was drawn. The cell activities were detected by MTT assay, and the absorbance values (A) were obtained by an enzyme mark instrument (SPMax 3500FL, Shanghai Flash Spectrum Biological Technology Co., Ltd.) at $490 \mathrm{~nm}$.

\section{CEA expression in the SW1116 cells transfected with pcDNA3.1(+)-DCC}

CEA expression was detected by immunofluorescence for $1,2,3$, and 4 days after transfection. Cells were suspended in $1 \mathrm{x}$ PBS and the concentration was $1 \times 10^{5}$ cells per milliliter. One milliliter cell suspension was absorbed into each tube, totaling $12 \times 75$ tubes. Mixture was centrifuged at $250 \mathrm{~g}$ for $5 \mathrm{~min}$ and supernatant was discarded. Primary antibody [CEA/CD66e (CB30) Mouse mAb \#2383, 1:1600] was added, mixed, and placed in tubes on ice for more than $30 \mathrm{~min}$ in the dark. Concentration was washed twice with $1 \mathrm{~mL}$ PBS and centrifuged for discarding the supernatant. The secondary antibody [Anti-mouse IgG $(\mathrm{H}+\mathrm{L})$, $\mathrm{F}\left(\mathrm{ab}^{\prime}\right) 2$ Fragment, Alexa Fluor ${ }^{\circledR} 488$ Conjugate, $\left.\# 4408,1: 1000\right]$ was added and mixed. Tubes were placed on ice for more than $30 \mathrm{~min}$ in the dark, washed twice with $1 \mathrm{~mL}$ PBS each tube and centrifuged for discarding the supernatant. One milliliter PBS was added and placed on ice in the dark. Cells were observed with the fluorescence microscope (TriM Scope, Lavision Biotec, Germany) and photographs were taken with the ImSpector Pro software.

\section{Statistical analysis}

Statistical analysis was performed using the SPSS version 15.0 software (SPSS, Chicago, IL, USA). Statistical analysis was conducted using a $t$-test. Differences at the level of P $\leq 0.05$ were considered to be statistically significant.

\section{RESULTS}

\section{Transfection with pcDNA3.1(+)-DCC decreased the proliferation and viability of SW1116 cells}

SW1116 cells were transfected with pcDNA3.1(+)-DCC and pcDNA3.1(+) vectors. Cells were detached with $0.25 \%$ trypsin and counted at $1,2,3,4,5$, and 6 days after transfection, and a growth curve was drawn (Figure 1). The results showed that the counts of SW1116 cells transfected with the pcDNA3.1(+)-DCC plasmid 3 to 6 days after transfection were significantly lower than those of cells transfected with the empty pcDNA3.1(+) vector and normal cell controls $(t 1=3.645, \mathrm{P} 1<0.05 ; t 2=3.132$, P $2<0.05$, Table 1$)$. Proliferation of the 
SW1116 cells transfected with pcDNA3.1(+)-DCC was also lower than that of cells transfected with pcDNA3.1 $(+)$ and of normal cell controls $(t 1=2.134, \mathrm{P} 2<0.05 ; t 2=2.736, \mathrm{P} 2<0.05)$. The cell viability was detected by MTT assay 1, 2, 3, 4, 5, and 6 days after transfection. Results from this assay suggested that the activity of the SW1116 cells transfected with pcDNA3.1(+)-DCC was lower than that of normal cell controls 2 to 6 days after transfection $(t 1=3.053, \mathrm{P} 1<0.05)$; in addition, the total cell activity of the SW1116 cells transfected with pcDNA3.1(+)-DCC was significantly lower than that of the control group of cells transfected with the empty plasmid $(t 2=$ $2.816, \mathrm{P} 2<0.05$, Table 2). Together, these results suggested that the proliferation of the colorectal cancer cell line SW1116 could be inhibited by transfection with the $D C C$ gene.

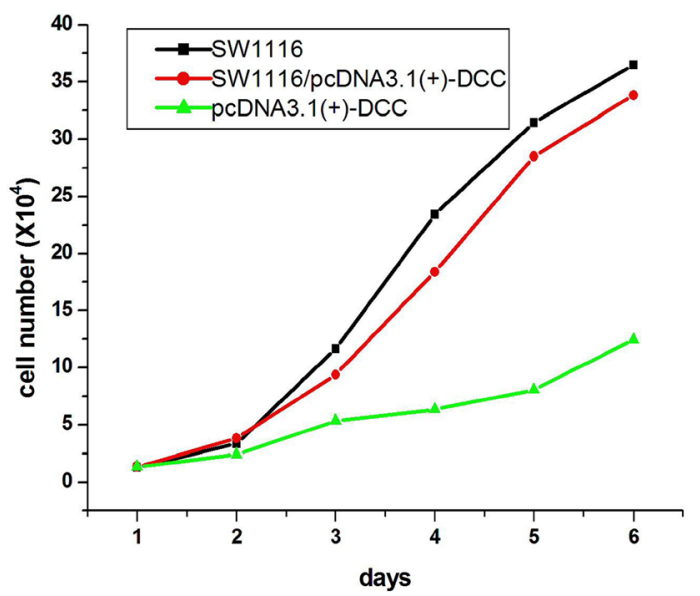

Figure 1. Growth curve of SW1116 cells transfected with the $D C C$ gene.

Table 1. Cell counts of the colorectal carcinoma cell line SW1116 transfected with the $D C C$ gene at different times.

\begin{tabular}{lcccccc}
\hline & \multicolumn{5}{c}{ Cell counts $\left(\mathrm{x} 10^{4}\right)$ (means \pm SD) } \\
\cline { 2 - 6 } & 1 day & 2 days & 3 days & 4 days & 5 days & 6 days \\
\hline Cell control & $1.30 \pm 0.11$ & $3.35 \pm 0.54$ & $11.66 \pm 1.53$ & $23.43 \pm 1.03$ & $31.45 \pm 0.79$ & $36.50 \pm 1.43$ \\
Transfected with empty vector & $1.37 \pm 0.46$ & $3.81 \pm 0.77$ & $9.38 \pm 1.00$ & $18.40 \pm 0.99$ & $28.51 \pm 1.04$ & $33.84 \pm 1.25$ \\
Transfected with DCC & $1.37 \pm 0.48$ & $2.43 \pm 0.45$ & $5.34 \pm 0.96$ & $6.34 \pm 0.99$ & $8.05 \pm 0.56$ & $12.45 \pm 1.45$ \\
Compared with cell control, P1 & 0.798 & 0.178 & $0.040^{*}$ & $0.003^{*}$ & $0.000^{*}$ & $0.005^{*}$ \\
Compared with vector control, P2 & 0.954 & $0.024^{*}$ & $0.000^{*}$ & $0.002^{*}$ & $0.000^{*}$ & $0.000^{*}$ \\
\hline
\end{tabular}

$* t 1=3.645, \mathrm{P} 1<0.05 ; t 2=3.132, \mathrm{P} 2<0.05 . \mathrm{SD}=$ standard deviation.

Table 2. Viability of SW1116 cells at different times after transfection with the $D C C$ gene, as detected using the MTT assay.

\begin{tabular}{lcccccc}
\hline & \multicolumn{4}{c}{$\mathrm{A}_{\text {490nm }}$ (means $\pm \mathrm{SD}$ ) } \\
\cline { 2 - 7 } & 1 day & 2 days & 3 days & 4 days & 5 days & 6 days \\
\hline Cell control & $0.34 \pm 0.03$ & $0.43 \pm 0.03$ & $0.53 \pm 0.02$ & $0.73 \pm 0.03$ & $0.85 \pm 0.04$ & $0.93 \pm 0.01$ \\
Transfected with empty vector & $0.34 \pm 0.02$ & $0.41 \pm 0.01$ & $0.51 \pm 0.01$ & $0.66 \pm 0.03$ & $0.81 \pm 0.04$ & $0.86 \pm 0.03$ \\
Transfected with the DCC gene & $0.33 \pm 0.04$ & $0.36 \pm 0.01$ & $0.44 \pm 0.05$ & $0.50 \pm 0.05$ & $0.57 \pm 0.02$ & $0.62 \pm 0.07$ \\
Compared with cell control, P1 & 0.838 & $0.041^{*}$ & $0.033^{*}$ & $0.003^{*}$ & $0.046^{*}$ & $0.015^{*}$ \\
Compared with vector control, P2 & 0.584 & $0.014^{*}$ & 0.159 & $0.007^{*}$ & $0.010^{*}$ & $0.023^{*}$ \\
\hline
\end{tabular}

$* t 1=3.053, \mathrm{P} 1<0.05 ; t 2=2.816, \mathrm{P} 2<0.05 . \mathrm{SD}=$ standard deviation; MTT $=$ methyl thiazolyl tetrazolium. 


\section{Transfection with pcDNA3.1(+)-DCC inhibited the expression of CEA in SW1116 cells}

SW1116 cells were transfected with either pcDNA3.1(+)-DCC or pcDNA3.1(+). The cells were fixed 4 days after transfection, and the CEA expression was detected by immunofluorescence staining. Results are shown in Figure 2: CEA appeared as yellowish green fluorescence under a UV lamp after immunofluorescence staining. The cell count showed that the number of yellowish green fluorescing cells and the fluorescence intensity of the SW1116 cells transfected with pcDNA3.1(+)-DCC were significantly lower than those of SW1116 cells transfected with pcDNA3.1(+) and of control cells. This result suggested that SW1116 cells transfected with the $D C C$ gene could downregulate their level of CEA expression.
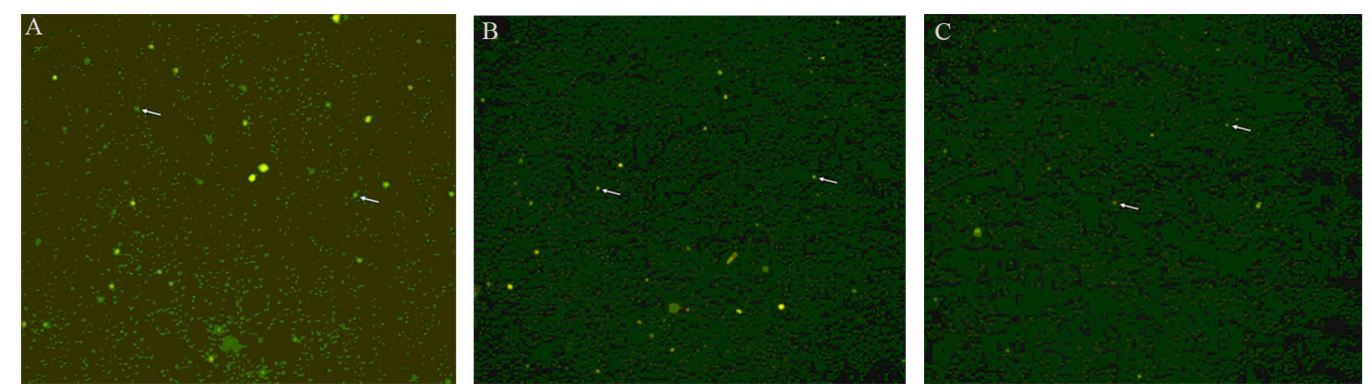

Figure 2. Immunofluorescence images of CEA expression in SW1116 cells transfected with the DCC gene (100X). A) Cell control; B) cells transfected with pcDNA3.1(+); and C) cells transfected with pcDNA3.1(+)-DCC. CEA, carcinoembryonic antigen.

\section{DISCUSSION}

DCC gene knockout mice supported HT-29 human colon cancer cell migration on nerve axons, but the differentiation of mucosal exocrine gland cells and tumors were inhibited, suggesting that the adhesion effects among cells might be improved and the relationship with the cell matrix might be decreased in HT-29 cells transfected with the DCC gene, potentially by destabilization of microsomes (Martin et al., 2010). $\beta$-catenin was identified as the main component of cell matrix adhesion, which is necessary to maintain connection among cells. When the Wnt signaling pathway is unimpaired, $\beta$-catenin is not degraded and transferred into the nucleus, where it can interact with lymphocyte enhancement factor and induce the transcription of a series of target genes. In the above experiment, it was found that the decrease of the connection between cells improved Wnt signaling and activated the migration progress. Recent studies have similarly suggested that tumor aggression and associated adverse results can be caused by the decrease of $\beta$-catenin expression and abnormalities in the Wnt signal pathway (Clevers, 2006).

$D C C$ gene knockout mice with colorectal cancer have also confirmed the important role of the focal adhesion kinase $(F A K)$ gene in tumor migration. The significance was different than that of mucus receptors. HT-29 human colorectal cancer cells transfected with the DCC gene appeared non-dispersed, and the amount of cadherin increased on the surface of cells (Dickson et al., 2002). The adhesion structure among cells and between cells and the matrix in different tissues has been shown to play a role in cellular migration required in the maintenance and development of normal structures. Ligands linked with the matrix and those of neighboring cells provide the molecular supports for biochemical pathways at the cell 
surface for cytoskeleton (Elliott et al., 2005). In fact, membrane receptors such as integrin, cadherin, selectin, and cell adhesion molecules were shown to be arranged under certain circumstances in clusters. Cytoskeletal proteins in the matrix were shown to be linked to those of neighboring cells, which play an especially important role in signal transduction in the human body, especially in the signals transduction (Forcet et al., 2002) in human body. The functional deletion of the immunoglobulin super family in colorectal cancer was taken as evidence that expression of this family of proteins was related to cell junctions, differentiation, apoptosis, and axonal guidance. Src (tyrosine kinase) and FAK of DCC in the DCC knowcout mice were shown to be phosphorylated, which was necessary for axonal rejection (Gautreau et al., 2002).

To address the potential of $D C C$ as a candidate for gene therapy in colon cancer, we first isolated and amplified a functional $D C C$ gene fragment. The $D C C$ functional fragments were obtained from normal human colon tissues using RT-PCR, and then were ligated with the pMD18-T vector and sequence verified. The sequence alignment of the sequencing result and the mRNA sequence of the DCC gene (accession No. NM_005215.3) reported in GenBank were conducted and shown to have complete identity. The pMD18-T-DCC was double digested with $X h o I$ and HindIII, and DCC gene fragments were recovered after agarose gel electrophoresis; simultaneously, the pcDNA3.1(+) vector was double digested by the same restriction endonucleases, and the vector fragments were recovered. Subsequently, ligation reactions of the $D C C$ gene fragment and pcDNA3.1 $(+)$ were conducted and the products were transformed into JM109 competent bacteria. Monoclonal colonies were picked, and positive recombination events were identified by enzyme digestion. The pcDNA3.1(+)-DCC recombinants were amplified and purified. Following transfection with the pcDNA3.1(+)-DCC plasmid, the count of SW1116 cells 3 to 6 days after transfection was significantly lower than that of cells transfected with the empty pcDNA3.1(+) vector or of normal cell controls $(\mathrm{P}<0.05)$. In addition, the proliferation of the SW1116 cells transfected with pcDNA3.1(+)-DCC was also lower than that of cells transfected with pcDNA3.1 $(+)$ and of normal cell controls $(\mathrm{P}<0.05)$. The cell activities were detected by MTT assay 1, 2, 3, 4, 5, and 6 days after transfection; the results suggested that the activities of the SW1116 cells transfected with pcDNA3.1(+)-DCC were lower than those of normal cell controls 2 to 6 days after transfection; furthermore, the total activities of the SW1116 cells transfected with pcDNA3.1(+)-DCC were significantly lower than those of the cells transfected with the empty plasmid at 2, 3, 4, 5, and 6 days after transfection. These results demonstrate that the proliferation of the colorectal cancer cell line SW1116 could be inhibited by transfection with the DCC gene. Transfection of SW1116 cells with the $D C C$ gene might inhibit its CEA expression. To examine whether this might occur, SW1116 cells were transfected with either pcDNA3.1(+)-DCC or pcDNA3.1(+), and the cells were fixed 4 days after transfection; CEA expression was detected by immunofluorescence staining. The results of this analysis are shown in Figure 2: positive CEA expression appeared as yellowish green fluorescence under a UV lamp after immunofluorescence staining. The cell counts demonstrated that the number of cells with yellowish green fluorescence and the fluorescence intensity of SW1116 cells transfected with pcDNA3.1(+)-DCC were significantly lower than those of SW1116 cells transfected with pcDNA3.1(+) and of control cells. The results suggested that SW1116 cells transfected with the DCC gene could downregulate their CEA expression. In addition, these results demonstrated that the DCC gene could be stably expressed in transfected cells after SW1116 transfection in vitro, which might provide an effective means for studying DCC function and its efficacy as a gene therapy agent for tumors. Thus, the results of our study suggest that DCC might be an effective target for gene therapy. 


\section{Conflicts of interest}

The authors declare no conflict of interest.

\section{ACKNOWLEDGMENTS}

Research supported by the Inner Mongolia Autonomous Region Natural Science Fund Project (\#2013MS1151).

\section{REFERENCES}

Clevers H (2006). Wnt/beta-catenin signaling in development and disease. Cell 127: 469-480.

Dard N, Louvet S, Santa-Maria A, Aghion J, et al. (2001). In vivo functional analysis of ezrin during mouse blastocyst formation. Dev. Biol. 233: 161-173.

Defacque H, Egeberg M, Habermann A, Diakonova M, et al. (2000). Involvement of ezrin/moesin in de novo actin assembly on phagosomal membranes. EMBO J. 19: 199-212.

Dickson TC, Mintz CD, Benson DL and Salton SR (2002). Functional binding interaction identified between the axonal CAM L1 and members of the ERM family. J. Cell Biol. 157: 1105-1112.

Elliott BE, Meens JA, SenGupta SK, Louvard D, et al. (2005). The membrane cytoskeletal crosslinker ezrin is required for metastasis of breast carcinoma cells. Breast Cancer Res. 7: R365-373.

Forcet C, Stein E, Pays L, Corset V, et al. (2002). Netrin-1-mediated axon outgrowth requires deleted in colorectal cancerdependent MAPK activation. Nature 417: 443-447.

Gautreau A, Louvard D and Arpin M (2002). ERM proteins and NF2 tumor suppressor: the Yin and Yang of cortical actin organization and cell growth signaling. Curr. Opin. Cell Biol. 14: 104-109.

Li PL, Hu CJ, Li CM, Meng CY, et al. (2006). Growth inhibitory effects of lipofectamine-mediated DCC gene on ovarian epithelial carcinoma. Zhonghua Fu Chan Ke Za Zhi 41: 186-189.

Martin M, Simon-Assmann P, Kedinger M, Martin M, et al. (2010). DCC regulates cell adhesion in human colon cancer derived HT-29 cells and associates with ezrin. Eur. J. Cell Biol. 85: 769-783. 